\title{
La solución de Contadora para el logro de la paz en Centroamérica
}

Una de las crisis internacionales más graves que encara el mundo actual es el conflicto en Centroamérica, cuya escalada podría conducir a una confrontación armada de proporciones. Urge hallar una salida, aunque no es fácil vislumbrar una solución viable, dada la complejidad de la situación.

Ha habido numerosas iniciativas de paz. Entre ellas el llamado proceso Gontadora destaca quizás como el único camino promisorio para lograr un arreglo negociado global. A su vez, su importancia trasciende los objetivos concretos de lograr una solución diplomática para la crisis. Señala nuevos rumbos en la política exterior de un grupo importante de países, que podria tener considerables repercusiones para el futuro, como en el caso de la cooperación regional latinoamericana.

\section{Los caminos que convergen a Contadora}

En enero de 1983 los ministros de relaciones exteriores de México, Colombia, Venezuela y Panamá se reunieron en la isla de Contadora, en el Golfo de Panamá, para examinar la situación en Centroamérica, analizar todas las propuestas de paz formuladas hasta entonces $\mathrm{y}$ proponer su propio plan para lograr una paz negociada en Ia región. Era evidente que por consideraciones de seguridad interna esos países tenían un interés común en la estabilización de la volátil situación en Centroamérica. Lo que constituyó una sorpresa fue la condenación explícita de la intervención extranjera en Centroamérica por los lideres del grupo Contadora y su convicción manifiesta de que las raíces del problema eran las desigualdades sociales y económicas en la región y que, por tanto, dichos problemas

* La autora es becaria investigadora en Chatham House y se especializa en América Latina. Es la editora de un volumen escrito por varios autores sobre América Latina y la recesión mundial que publicará en 1985 Ia Cambridge University Press y el Royal Institute of International Affairs. El presente artículo aparece en forma simultánea en alemán en Europa-Archiv (Bonn). 
no deblan situarse dentro del contexto del conflicto Este-Oesté. Esta posición, adoptada por cuatro destacados paises de la región, fue un severo golpe para Washington, que buscaba el consenso regional para sus políticas y enfoques con respecto a Centroamérica.

Para tres de los países participantes en Contadora (Colombia, Venezuela y Panamá), esta actitud significaba apartarse de la posición previa de apoyo a las políticas estadounidenses en Centroamérica. También para México significó apartarse de posiciones previas, al menos en cuanto a estilo y estrategia. ¿Cuáles fueron las causas de dichos cambios?

Ya durante la administración de López Portillo, la actitud de México hacia Centroamérica había sido radical. Esta se reflejó en el comunicado franco-mexicano sobre El Salvador, emitido en agosto de 1981, que reconocía la legitimidad de las fuerzas insurgentes en El Salvador; en la decisión de México de ponerse de parte de Nicaragua y Granada contra la resolución aprobada por la Organización de Estados Americanos (OEA) Dara acoger con beneplácito el anuncio formulado por EI Salvador de celebrar elecciones en marzo de 1982; y en el decisivo apoyo moral y material de México al régimen sandinista. Este radicalismo habla significado no sólo conflictos con los Estados Unidos sino que había aislado a México de sus vecinos latinoamericanos.

Hubo dos acontecimientos que modificaron la posición de México frente a Centroamérica: uno fue la crisis financiera del verano boreal de 1982, en que los Estados Unidos se apresuraron a servir de fiadores; y el otro, la asunción de un nuevo Presidente, Miguel de la Madrid, cuyo Canciller, Bernardo Sepúlveda, tenía un estilo diplomático más sobrio y "clásico" que su predecesor. Aunque el nuevo gobierno declaró que la crisis financiera no alteraría las poIfticas de México respecto de Centroamérica, se podfan advertir algunas diferencias. De una política radical, casi agresiva, México pasó a una actitud más discreta; de un papel de liderazgo solitario se inclinó hacia uno colectivo; y de la búsqueda de legitimación por parte de potencias extrarregionales (como Francia) pasó a un acercamiento con sus pares regionales latinoamericanos.

Sin embargo, hay un elemento importante de continuidad en las dos administraciones: la función de "comunicador" entre Ios Estados Unidos y lo que este último considera como las fuentes de la subversión en Centroamérica, a saber Guba, Nicaragua y la oposición salvadoreña. México ha seguido desempeñando este papel importante durante el gobierno de De la Madrid. De hecho, la nueva administración se ha apuntado varios éxitos por este concepto. Los más recientes fueron la visita del Secretario de Estado, George Shultz, a Managua, poco después del viaje del Presidente De la Madrid a Washington en mayo $y$, luego, las conversaciones celebradas en Manzanillo entre el enviado especial estadounidense para Cen- 
Esperanza Dusán / La solución de Contadora para el logro de la paz...

troamérica, Harry Shlaudeman, y el Viceministro de Relaciones Exteriores nicaragüense, Víctor Tinoco. Ambas iniciativas fueron negociadas por De la Madrid como parte de sus esfuerzos por lograr un mejoramiento de las relaciones entre Estados Unidos y Nicaragua.

Para desempeñar su nuevo papel pionero en Centroamérica, México necesitaba hallar partícipes del mismo parecer. En 1983 ya los había encontrado. Había llegado el momento para que surgiera Contadora. Varios acontecimientos habían aglutinado a Venezuela, Colombia y Panamá en torno al enfoque mexicano.

En Venezuela, la naturaleza democrática de su sistema había sido la fuente principal de cambio en su política exterior. Bajo la presidencia social demócrata de Carlos Andrés Pérez, Venezuela había ayudado activamente a los sandinistas a conquistar el poder mediante el otorgamiento de ayuda política y económica. Cirando $\mathrm{He}$ rrera Campins sucedió a Pérez, Ia ayuda se desvió de Nicaragua hacia el gobierno de Napoleón Duarte, en EI Salvador, amigo personal y colega democratacristiano del nuevo Presidente. Campins contribuyó además con asistencia militar y adiestramiento para el ejército salvadoreño. Fue durante la administración de Campins que Venezuela comenzó a respaldar la política estadounidense hacia EI Salvador y respecto a Centroamérica en general. Venezuela desempeñó un papel rector en la oposición contra el comunicado franco-mexicano y apoyó a la Comunidad Democrática Centroamericana (CDC), cuya formación fue inspirada por Estados Unidos, pasando a ser un observador en la misma.

Hubo dos acontecimientos externos que provocaron un cambio en la política centroamericana de Venezuela. Primero, la derrota de Duarte en las elecciones de marzo de 1982 entibió el apoyo venezolano a EI Salvador; por ende, ya no estaba presente el elemento personal y partidista que explicaba las buenas relaciones entre ambas naciones. Poco después estallaba el conflicto del Atlántico Sur, y el papel de los Estados Unidos como fiel partidario del Reino Unido hizo que Venezuela lo pensara dos veces antes de ayudar a los Estados Unidos a lograr sus objetivos en Centroamérica.

Los primeros indicios de cambio en la actitud venezolana se produjeron en septiembre de 1982, cuando Venezuela se unió a México para impulsar una iniciativa de paz en que ambos paises ofrećan su mediación para evitar un conflicto armado entre Nicaragua y Honduras.

Hasta entonces, México y Venezuela, apoyados por su riqueza petrolera, eran decididamente los actores políticos y diplomáticos latinoamericanos más importantes que tomaban las iniciativas principales con respecto a Centroamérica. Pero el surgimiento de un nuevo participante activo e independiente en Centroamérica ocurrió a 
mediados de 1982 con la elección de Belisario Betancur como Presidente de Colombia.

En el gobierno de Turbay Ayala, predecesor de Betancur, Colombia había apoyado las políticas de Reagan en la zona. Colombia había aceptado convertirse en el quinto miembro del Grupo Nassau y contribuir a esta forma inicial multilateral de la Iniciativa para la Cuenca del Caribe (Garibbean Basin Initiative-CBI) ${ }^{1}$; había respaldado a la cDC, y había participado también como miembro fundador en el Foro para la Paz y la Democracia en San José. EI alineamiento de Golombia con Ios Estados Unidos no obedecía sólo a la similitud de criterios de Turbay y Reagan con respecto a Centroamérica, sino también al hecho de que Colombia había recibido una ayuda militar considerable de los Estados Unidos. Y lo que es más importante, el gobiemo de Reagan había respaldado a Colombia cuando el régimen sandinista de Nicaragura había reactivado sus reclamaciones de soberanía sobre las islas colombianas de San Andrés y Providencia, situadas frente a la costa nicaragüense.

Esta tendencia pro-Washington se invirtió al asumir el poder Betancur. La nueva administración introdujo grandes cambios en la política interior y exterior. Por ejemplo, ofreció amnistía a todos los grupos guerrilleros en Colombia. Procuró mejorar las relaciones con Cuba, solicitó su ingreso al Movimiento no Alineado y le dio gran importancia a las relaciones exteriores. Contadora ofrecfa una buena oportunidad para poner en práctica estas aspiraciones.

A primera vista, la participación de Panamá en el Grupo Contadora parecería algo inesperada. Panamá había adoptado tradicionalmente una actitud flexible en materia de polftica exterior en general, y en cuanto al conflicto centroamericano en particular, habfa permanecido relativamente tranquilo. Sin embargo, tal como ocurrió con Venezuela, fue el conflicto de las Malvinas y la posición adoptada por los Estados Unidos al respecto lo que contribuyó a que Panamá reevaluara su propio papel en la región y sus relaciones con los Estados Unidos.

Panamá pasó a tomar parte activa en Centroamérica en el verano boreal de 1982 con la presentación de un plan de paz que proponfa un sistema de pactos de no agresión entre Nicaragua y los Estados Unidos y entre Nicaragua y la cDc; una solución negociada para la guerra civil en El Salvador; y el comienzo de un proceso de distensión entre los Estados Unidos y Cuba. La propuesta panameña incluía asimismo garantías de soberanía territorial para todas las naciones centroamericanas a fin de impedir incursiones armadas desde países vecinos. Esta iniciativa de paz no prosperó, pese a es-

"Véase, Ramesh Ramsaran, "The us Caribbean Basin Initiative", The World Today, noviembre de 1982. 
tar respaldada por Colombia, Costa Rica, Honduras, Venezuela y Nicaragua.

Otras dos tentativas de Panamá para contribuir a la pacificación de la zona fracasaron. Una en noviembre de 1982 cuando el Presidente De la Espriella visitó MIéxico, El Salvador y Nicaragua, ofreciéndose para oficiar de mediador en las negociaciones entre el Gobierno de EI Salvador y la oposición. La otra fue la sugerencia de De la Espriella de que Felipe González, Primer Ministro de España, participara para contribuir a Ios planes de paz de la región.

Era evidente que la estatura de Panamá en los asuntos internacionales era insuficiente para reunir apoyo y tener la influencia necesaria para ser tomado en serio. Contadora ofrecía la mejor alternativa para actuar en forma más eficaz.

\section{Contadora tras 18 meses}

Desde su establecimiento en 1983, el Grupo Contadora ha ofrecido nuevas esperanzas de una solución diplomática para el conflicto en Centroamérica. Como sus credenciales regionales eran impecables, se creyó en su deseo de actuar en forma imparcial.

El Grupo Contadora procuró establecer un marco dentro del cual pudieran realizarse negociaciones y ofrecer también un foro permanente para el diálogo entre los países centroamericanos. Se seleccionaron varios temas claves que podian ser materia de negociaciones. Éstos comprendían: el retiro de todos los asesores militares extranjeros, el cese de las ventas de armamentos y la congelación del nivel de armamentos existente, la ejecución de un plan de reactivación económica y la solución de los problemas internos por métodos democráticos, con la inclusión de todos los grupos políticos en cada pafs.

Durante 1983 los esfuerzos de Contadora se vieron entorpecidos - por varios obstáculos, como la actitud belicosa de la administración Reagan contra el régimen sandinista de Nicaragua, la desconfianza entre Ios estados centroamericanos, y una verdadera abulia para proseguir las negociaciones. Honduras, EI SaIvador y Costa Rica desconfiaban de la veracidad de las intenciones profesadas por Nicaragua de no exportar la revolución; de su ofrecimiento de desmilitarizarse y su promesa de democratización y de celebrar elecciones libres. Por su parte, Nicaragua se mostraba escéptica acerca de la buena disposición de sus vecinos para prescindir de los asesores militáres extranjeros y de la ayuda militar que recibian de Ios Estados Unidos.

En la séptima reunión del Grupo Gontadora celebrada en septiembre de 1983, se establecieron tres grupos de trabajo encargados de aspectos de seguridad, políticos y socio-económicos. De esta reunión emanó el logro más importante de Contadora en el primer 
año de su creación: un programa de 21 puntos para garantizar la paz, la seguridad y la estabilidad de la región. El programa, cuyos elementos claves comprendían la congelación de las importaciones de armas y el tamaño de los ejércitos, la reducción del número de asesores militares extranjeros, y un pacto de no agresión que prohibía la utilización de los territorios nacionales para atacar a otros países, fue apoyado por los cinco países centroamericanos. El Presidente Reagan convino en que el documento de 21 puntos era la base mejor para lograr una solución perdurable de los problemas de Centroamérica.

Se pretendía que el programa de 21 puntos sirviera de base para elaborar un tratado de paz obligatorio para las cinco naciones de Centroamérica que se firmarła en diciembre de 1983. Pero hubo que postergar la reunión para la firma del tratado porque Honduras, El Salvador, Guatemala y Costa Rica se opusieron a las adiciones que Nicaragua exigla que se introdujeran en el programa. Estos países temían que si aceptaban estas exigencias adicionales nicaragüenses dejarian intacta la capacidad militar de Nicaragua, mientras se forzaba la eliminación de las bases militares que los Estados Unidos tienen en otros países ${ }^{2}$.

Pese a este revés, los Cancilleres del Grupo Contadora y de los cinco países centroamericanos volvieron a reunirse a comienzos de este año y llegaron al acuerdo preliminar de preparar un tratado de paz definitivo, dentro de seis meses.

En esa etapa, después de quince meses de negociaciones, reuniones, propuestas y contrapropuestas, el limado de algunas aristas para lograr un acuerdo, parecía dudoso el éxito de 'Contadora e incluso su propia existencia. En una reunión celebrada en Panamá en abril para evaluar la marcha de los trabajos, los Cancilleres de Contadora observaron que las fuerzas irregulares eran apoyadas $y$ abastecidas por países vecinos; que estaban ocurriendo algunas actividades, como el minado de puertos, que perturbaban el comercio y. la libertad de navegación; que se había intensificado la tensión y que aumentaba la desconfianza, como resultado de la presencia creciente de tropas extranjeras y de asesores militares extranjeros; y que había aumentado el nivel de los armamentos y la proliferación

${ }^{2} \mathrm{La}$ propuesta nicaragüense, que había sido rechazada por Washington, consistia en la firma de cuatro tratados: dos entre los Estados Unidos y Nicaragua que prohibian el establecimiento de bases militares extranjeras en Centroamérica y garantizaban el libre tránsito de los barcos y aviones estadounidenses por las aguas y el espacio aéreo nicaragüense; un tratado regional para los cinco estados centroamericanos que prohibia el tráfico de armas, así como las acciones manifiestas o encubiertas destinadas al derrocamiento de gobiernos establecidos, (incluido el apoyo logistico a fuerzas irregulares en la region); y un tratado sobre El Salvador, que involucraría a todas las partes y países en la solución del conflicto en esa nación. 
de las maniobras militares. En suma, los llamamientos de Contadora no habían surtido efecto.

Sin embargo, Contadora ha sobrevivido y ha llegado a la culminación de sus esfuerzos en pro de la paz con la redacción, a comienzos de junio, de la llamada Acta de Contadora. Es un plan de paz elaborado en forma de tratado que sería obligatorio para los cinco signatarios centroamericanos. (Su texto aún no se ha dado a la publicidad, pero este autor conoce su contenido).

El Acta de Contadora representa un esfuerzo por armonizar todas las propuestas que han surgido desde que se inició el proceso. Subraya la necesidad de la cistensión en Centroamérica y la importancia de las medidas generadoras de confianza. Estipula las normas para la realización de maniobras militares, prohíbe la creación de nuevas bases militares extranjeras o de establecimientos de adiestramiento, y propone Ia eliminación de los existentes dentro de un año de firmado el tratado; afirma la necesidad de poner fin a la venta de armamentos, al apoyo de fuerzas irregulares y a la carrera de armamentos en la zona; y lo que es más importante, propone el establecimiento de un mecanismo de control y verificación mediante una comisión permanente y autónoma.

Para facilitar la coordinación, el Acta prevé la creación de canales de comunicación rápidos entre los gobiernos y las autoridades militares y el establecimiento de comisiones mixtas de seguridad, en particular entre Honduras y Nicaragua, y El Salvador y Nicaragua, y el fortalecimiento de las existentes, como la comisión Costa Rica-Nicaragua (restablecida en mayo de 1984 bajo los auspicios de Contadora).

En la esfera política, el Acta de Contadora defiende Ios principios del pluralismo democrático con plena libertad para las diferentes corrientes de opinión, la reconciliación nacional y el diálogo, que incluiría garantías para los opositores y amnistiados. Se prevé la formación de una comisión política, en forma similar a la militar, para que reciba y evalúe la información sobre cómo se cumplen las obligaciones en materia de derechos políticos, electorales y humanos contraidos por los signatarios.

\section{Perspectivas de Contadora}

¿Cuáles son las posibilidades de que un tratado de paz global de carácter jurídico - como el Acta de Contadora- sea aceptado y ratificado por las cinco repúblicas centroamericanas? Antes de evaluar las perspectivas de que los países centroamericanos acepten firmar dicho tratado, convendría referirse someramente a los dos paises principales situados fuera de la región, que tendrían inevitablemente que desempeñar un papel rector en todo proceso serio de paz en Centroamérica, es decir, Cuba y los Estados Unidos. 
Cuba ha desempeñado un papel importante en la región, pero lo ha hecho de manera más bien discreta. Aparte de pronunciamientos ocasionales de escasa significación y de un respaldo formal a la posición mexicana antes y después de Contadora, Cuba ha eludido la atención general. Esto puede ser un reflejo del repliegue general de Cuba después de sus aventuras militares en Africa y su desprestigio diplomático más reciente en el asunto de Grana$\mathrm{da}^{3}$. Sin embargo, no debe subestimarse su papel indirecto. El origen de la intransigencia, la desconfianza y la suspicacia nicaragüense proviene de Cuba.

Sentimientos similares han caracterizado al gobierno de Reagan, el que achaca la inestabilidad en Centroamérica al expansionismo soviético y cubano. La "teoría del dominó" ha cautivado la mente de los formuladores de política del gobiernot.

La actitud de Reagan hacia Centroamérica no se ha ablandado, pero ha habido cierta flexibilidad táctica. Por ejemplo, por una parte se ha incrementado la presión militar sobre los rebeldes salvadoreños y el régimen sandinista, pero por otra, ha habido también iniciativas diplomáticas como la celebración de conversaciones con las guerrillas en El Salvador o el apoyo al proceso de Contadora. Cuando las dificultades con el Congreso han puesto en peligro el flujo de fondos para proseguir una política beligerante, el gobierno de Reagan ha recurrido a maniobras tales como el establecimiento de la comisión bipartita Kissinger, la designación de enviados especiales a Centroamérica o, en forma más reciente, la visita de Shultz a Managua. Los críticos del gobierno no pueden acusarlo de intransigencia, al menos en apariencia.

La conducta de la política estadounidense hacia Centroamérica ha dependido, asimismo, de qué parte del gobierno ha predominado en su formulación: Ios ideólogos de la línea dura, como la señora Jeane Kirkpatrick y aquellos que se ocupan de Centroamérica en la Casa Blanca, eI Consejo de Seguridad Nacional y la Agencia Central de Inteligencia (CTA), o los pragmáticos del Departamento de Estado ${ }^{5}$. Hasta hace poco, parece que eran los ideólogos los que predominaban. Sin embargo, al aproximarse las elecciones de noviembre ha habido una presión creciente sobre Reagan para demostrar que Ios Estados Únidos controlan el problema centroamericano $^{6}$.

\footnotetext{
"Véase, Tony Thorndike, "The Grenada crisis", The World Today, diciembre de 1983.

"Gordon Connel-Smith, "The crisis in Central America: Presidente Reagan's options", ibid., octubre de 1983.

"Barry Rubin, "Reagan administration policymaking and Central America", en Robert S. Leiken, Central America. Anatomy of Conflict (Nueva York: Pergamon Press, 1984), pp., 299-318.

Mark Falcoff, "The apple of discord: Gentral America in us domestic poli-
} 
Esta consideración puede haber conducido a abandonar la política de mantener a Shultz fuera de Centroamérica. La visita de Shultz a Managua el $1^{\circ}$ de junio significó apartarse bastante de la política estadounidense tradicional. Shultz fue el primer funcionario estadounidense de alto rango que visitó Managua después de la victoria sandinista en 1979.

Pero el establecimiento de un diálogo entre los Estados Unidos y Nicaragua por intermedio de la visita de Shultz a Managua y las conversaciones Shlaudeman-Tinoco sostenidas en Manzanillo, pueden haber tenido otras razones aparte de las necesidades electorales de Reagan. La reacción internacional y, sobre todo, la reacción nacional ante el minado de los puertos nicaragüenses por los Estados Unidos puede haber inducido a Reagan a percatarse de que esto podia crearle problemas en el Congreso y poner en peligro todo el paquete de ayuda destinado a El Salvador, también argumentado en el informe de la comisión bipartita.

En Nicaragua, Ios sandinistas tienen razones muy poderosas para mostrar cierta flexibilidad antes de las elecciones de noviembre en los Estados Unidos. Por ahora, pueden ejercer cierta influencia sobre el gobierno de Reagan y podrían llegar a un compromiso con los Estados Unidos antes de noviembre. También podrían hacer un gran esfuerzo para conseguir la firma del acta de Contadora. Pasado noviembre no les quedará poder de negociación a los sandinistas. Si Reagan es reelegido, lo que parece muy probable, su gobierno podría endurecer su actitud, esta vez con legitimación electoral, y tendría cuatro años para poner en práctica con mayor libertad políticas en estricta consonancia con su ideología, donde los sandinistas no cuentan.

Por cierto que el papel de los demás países centroamericanos también es decisivo para el éxito de todo acuerdo de pạz perdurable. Comparten ciertos intereses y tienen la percepción común de que el poderío militar de Nicaragua constituye una grave amenaza para su propia seguridad. En este sentido necesitan encontrar un bastión contra él y por eso confían en la ayuda militar de Washington. Fueron el temor de la militarización de Nicaragua y la presión de Washington lo que condujo a Honduras, El Salvador y Guatemala a reactivar el pacto militar de 1965 , denominado CONDECA (Consejo de Defensa Centroamericano) después de que el proceso de Contadora se había iniciado.

Otra inquietud que compartían Honduras, Costa Rica y Guatemala (para qué decir de El Salvador), era la posibilidad de una victoria militar de los insurgentes en EI Salvador, lo que consideraban

tics", en Howard J. Wiarda, Rift and Revolution. The Central American imbroglio. (Washington: American Enterprise Institute, 1984), pp. 360-82. 
como un absoluto desastre. Para esos países, un elemento positivo que contribuye a la estabilidad de la región es la victoria reciente de Duarte sobre su oponente de extrema derecha, Roberto d'Aubuisson, en la elección de dos rueltas celebrada en El Salvador. ConDuarte firmemente asentado en el poder, toda ayuda al gobierno de El Salvador ya sea de los Estados Unidos o de otra parte, en caso de que las guerrillas recobraran su fuerza, se torna ahora respetable y garantizará la imposibilidad de un éxito militar para los insurgentes. Tal vez este sea un elemento a favor del apoyo pleno al Acta de Contadora, a fin de consolidar lo ganado en El Salvador.

El papel de Costa Rica en participar es de suma importancia en esta coyuntura. Su carencia de ejército, su neutralidad, y el nuevo papel activo asumido por el Presidente Monge en la pacificación. de la región convierte a Costa Rica en el agente ideal para persuadir a las naciones más inflexibles de Centroamérica - Honduras y Guatemala- a que opten por una solución diplomática. La reciente gira europea del Presidente Monge para obtener apoyo en pro de la paz en Centroamérica y la reunión que se celebrará en Costa Rica en septiembre de los Ministros de Relaciones Exteriores de los principales países de Europa, los cuatro de Contadora y los cinco de Centroamérica son indicios evidentes de la intención de Costa Rica de proseguir el camino hacia una solución diplomática.

\section{Contadora: ¿̨éxito o fracaso?}

El proceso de Contadora ha conseguido algunos resultados, pero no su objetivo esencial: la solución diplomática del conflicto centroamericano. Algunos han sostenido que después de 18 meses Contadora tiene poco que mostrar en cuanto a resultados concretos. Sin embargo, sería injusto y prematuro juzgar en esta forma el éxito relativo de Gontadora. Más bien, los resultados deberian medirse tomando en cuenta los inconvenientes que ha encarado desde su creación.

Su papel parece bastante más constructivo si se toman en consideración algunos "intangibles". Primero, logró definir los aspectos claves que son requisitos esenciales para la estabilización de la región. Segundo, pese a diferencias considerables entre sus miembros, Contadora ha logrado alcanzar un consenso regional respecto a la forma y los medios de abordar los problemas más urgentes. Tercero, ha reunido a las circo naciones centroamericanas directamente involucradas para que ventilen sus quejas. Aunque no se han logrado progresos reales para eliminar la desconfianza entre los países de la región, las ventajas del establecimiento de un diálogo y de una base para el análisis son innegables $y$ pueden ser de gran valor en 
Esperanza Duran / La solución de Contadora para el logro de la paz...

el futuro. Cuarto, aunque esto todavía no puede demostrarse, Contadora habría servido para aliviar y contener la tensión en la región en un momento de grave inestabilidad, al borde de una conflagración en gran escala. 